\section{Cirurgia para diminuir a freqüência fundamental da voz - tireoplastia tipo III de Isshiki}

\author{
Domingos H. Tsuji ${ }^{1}$, Luiz U. Sennes ${ }^{2}$, \\ Rui Imamura ${ }^{3}$, Priscila M. Trezza ${ }^{4}$, \\ Eliana M. Hanayama ${ }^{5}$
}

Resumo

\begin{abstract}
A vOZ aguda em excesso pode constituir verdadeiro problema social e profissional, principalmente quando peculiar a indivíduos do sexo masculino. Suas causas, em geral, são orgânicas e/ou funcionais. A causa funcional mais freqüentemente encontrada é o distúrbio conhecido como falsete mutacional, cuja terapia de primeira escolha é a vocal. Entretanto, quando seus resultados não são satisfatórios, o tratamento cirúrgico é uma opção muito conveniente e eficaz. Apresentamos dois casos de pacientes do sexo masculino, de 34 e 35 anos de idade, com voz demasiadamente aguda, resistentes à fonoterapia, que foram tratados com sucesso pela tireoplastia tipo III de Isshiki. A técnica cirúrgica, assim como a história clínica e os achados vocais pré e pós-operatórios são apresentados com detalhes neste trabalho. Expomos e discutimos, também, dados pertinentes ao tema encontrados na literatura. Concluímos que a tireoplastia tipo III de Isshiki é um procedimento eficaz, simples e com baixo índice de complicações, que pode ser utilizado para reduzir o pitch vocal em casos selecionados. Este estudo demonstrou dados que podem subsidiar educadores da área médica quando da reformulação do currículo de ORL para o curso de graduação em medicina.
\end{abstract}

Suligery to lower vocal pitch - Isshilki type III thyroplasty
Palavras-chave: distúrbios da voz, cirurgia, humano. Key words: voice disorders, surgery, human.

\begin{abstract}
$\mathrm{H}$ igh-pitched voice may cause social and professional embarrassment, particularly if it occurs in men. This disorder may be organic and/or functional. Mutational falsetto (puberphonia) is the most common functional cause and is primarily treated with phonotherapy. However, when results of this therapy are limited, surgery may turn to be a convenient and effective alternative to treat these patients. In this study, we present 2 male patients ( 34 and 35 years old) with high-pitched voices, who failed phonotherapy and who were successfully treated with Isshiki type III thyroplasty. Medical history of the patients, surgical techniques, pre and postoperative laringostroboscopies and outcomes are presented in detail. We conclude that Isshiki type III thyroplasty is an efficient, simple to perform and lowrisk procedure that may be used in selected patients with high-pitched voice.
\end{abstract}

\footnotetext{
${ }^{1}$ Médico Assistente Doutor da Divisão de Clínica Otorrinolaringológica do Hospital das Clínicas da Faculdade de Medicina da Universidade de São Paulo. ${ }^{2}$ Professor Doutor da Disciplina de Otorrinolaringologia da Faculdade de Medicina da Universidade de São Paulo.

3 Médico Assistente da Divisão de Clínica Otorrinolaringológica do Hospital das Clínicas da Faculdade de Medicina da Universidade de São Paulo e Doutorando da área de Otorrinolaringologia do Curso de Pós-Graduação da Faculdade de Medicina da Universidade de São Paulo.

Fonoaudióloga da Divisão de Clínica Otorrinolaringológica do Hospital das Clínicas da Faculdade de Medicina da Universidade de São Paulo e especialista em voz pelo Centro de Estudos da Voz - CEV - São Paulo.

${ }^{5}$ Fonoaudióloga com especialização pela Universidade de Kyoto, Japão e Professora do Curso de Pós-Graduação em Motricidade e Voz do CEFAC - São Paulo.Divisão de Clínica Otorrinolaringológica do Hospital das Clínicas da Faculdade de Medicina da Universidade de São Paulo.

Endereço para correspondência: Dr. Domingos H. Tsuji, R. Peixoto Gomide, 515, conj. 145, São Paulo - 01409-001

Tel: (0xx11) 251-5504 - E-mail: dtsuji@attglobal.net

Artigo recebido em 18 de maio de 2001. Artigo aceito em 17 de julho de 2001
} 


\section{INTRODUÇÃO}

A condição vocal de uma pessoa depende de diversos fatores, como a qualidade do som fundamental produzido na laringe, a ressonância sofrida por este durante sua propagação pelo trato vocal e a articulação desse último na região do palato, da língua, dos dentes e dos lábios. Essas características são particulares e exercem uma forte influência no meio social como importante fator de identificação individual. Quando apresenta um desvio muito grosseiro do padrão considerado normal, pode tornar-se extremamente inconveniente ao portador, produzindo forte impacto no comportamento psicológico e social do mesmo ${ }^{1}$. Uma das características vocais primordiais é aquela que depende da freqüência fundamental produzida na laringe, a qual tem forte correlação com o sexo: em indivíduos masculinos, ela costuma ser mais baixa, em torno de $113 \mathrm{~Hz}$, enquanto entre as mulheres é mais elevada, por volta de $205 \mathrm{~Hz}^{2}$. Indiscutivelmente, quando uma pessoa produz um pitch vocal não condizente com o de seu sexo, esse fator acaba por trazer muitos inconvenientes, principalmente em termos sociais, podendo causar inúmeros prejuízos psicológicos e profissionais. Dos distúrbios vocais, um dos mais freqüentemente observados em relação à incompatibilidade entre o pitch vocal e o sexo é aquele em que um homem apresenta voz excessivamente aguda. Essa condição é comumente conhecida como falsete mutacional ou muda vocal incompleta, que é rara, de origem predominantemente funcional e na maioria dos casos regride com terapia vocal, quando esta é bem conduzida. Em ocasiões incomuns, entretanto, a fonoterapia pode não surtir o resultado esperado, sendo necessária a intervenção cirúrgica. Uma das técnicas mais empregadas para abaixar o pitch vocal é a tireoplastia tipo III de Isshiki ${ }^{4,11,13}$, que consiste na redução da distância ântero-posterior das pregas vocais, obtida pela remoção de uma fita vertical da lâmina da cartilagem tireóidea. Com isso, a distância entre o processo vocal da aritenóide e o ponto de fixação da prega vocal na comissura anterior é encurtada, relaxando essa última, e promovendo a redução da freqüência vocal. O objetivo deste trabalho é o de apresentar a técnica da tireoplastia tipo III proposta por Isshiki, exemplificada por meio de dois casos cujo tratamento cirúrgico foi bem sucedido.

\section{TÉCNICA CIRÚRGICA}

Segundo Isshiki ${ }^{4,7,8,10}$, as cirurgias do arcabouço laríngeo classificam-se em quatro tipos de tireoplastias, quais sejam:

1) tipo I, para medialização da prega vocal - promovendo melhor fechamento glótico;

2) tipo II, para lateralização - reduzindo a resistência glótica;
3) tipo III, para relaxar as pregas vocais - abaixando o pitch vocal;

4) tipo IV, para tensionar ou estirar as pregas vocais elevando o pitch vocal.

O pitch vocal, sabidamente, depende de três fatores: massa, tensão e comprimento da prega vocal. A tireoplastia tipo III promove o encurtamento ântero-posterior da lâmina da cartilagem tireóidea, reduzindo a tensão das cordas vocais e aumentado a massa relativa da mucosa vibrátil. A cirurgia é feita sob anestesia local e sedação endovenosa, com o paciente em decúbito dorsal, tendo um coxim sob seus ombros para hiperestender o pescoço, o que facilita a exposição e a identificação das estruturas cartilaginosas da laringe.

Primeiramente, a linha de incisão transversal é demarcada na pele. Em geral, tem cerca de $5 \mathrm{~cm}$ de comprimento e situa-se no ponto médio do espaço entre a projeção da incisura superior e a borda inferior da cartilagem tireóidea (Figura 1). Em seguida, cerca de $4 \mathrm{ml}$ de xilocaína são injetados sob a pele, no local previamente demarcado. Subseqüentemente, a incisão é feita com bisturi no 15, expondo-se os músculos pré-laríngeos que podem, se necessário, ser total ou parcialmente seccionados de modo transversal. A linha mediana da cartilagem tireóidea é identificada e suas duas lâminas são expostas pelo menos em seus $2 / 3$ anteriores. A fita de cartilagem que deve ser removida é demarcada, aproximadamente, na linha de transição entre o terço anterior e o médio da lâmina da cartilagem tireóidea (Figura 2). Essa fita deve ter inicialmente cerca de $3 \mathrm{~mm}$ de largura. As incisões podem ser feitas com bisturi nº 11, ou com broca de aço Vanádio PM nº 38, de uso odontológico, e micro-motor. O pericôndrio externo não é descolado, sendo, portanto, seccionado durante a incisão da cartilagem. O pericôndrio interno, porém, deve

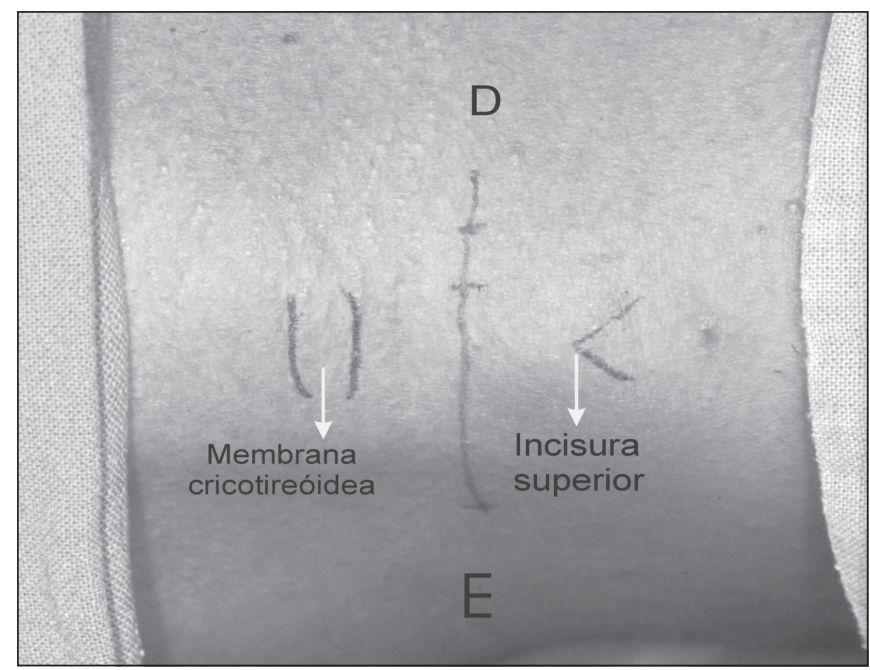

Figura 1. A incisão cervical situa-se no ponto médio da distância entre a incisura superior e borda inferior da cartilagem tireóidea. 


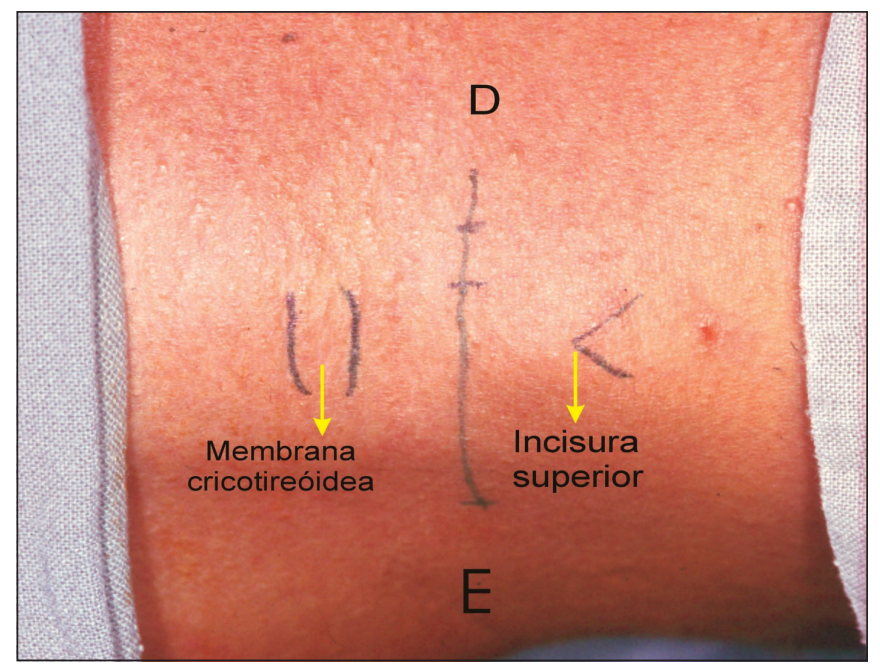

Figura 2. Uma fita de $3 \mathrm{~mm}$ de largura é demarcada entre o terço anterior e médio da lâmina da cartilagem tireóidea de um dos lados.

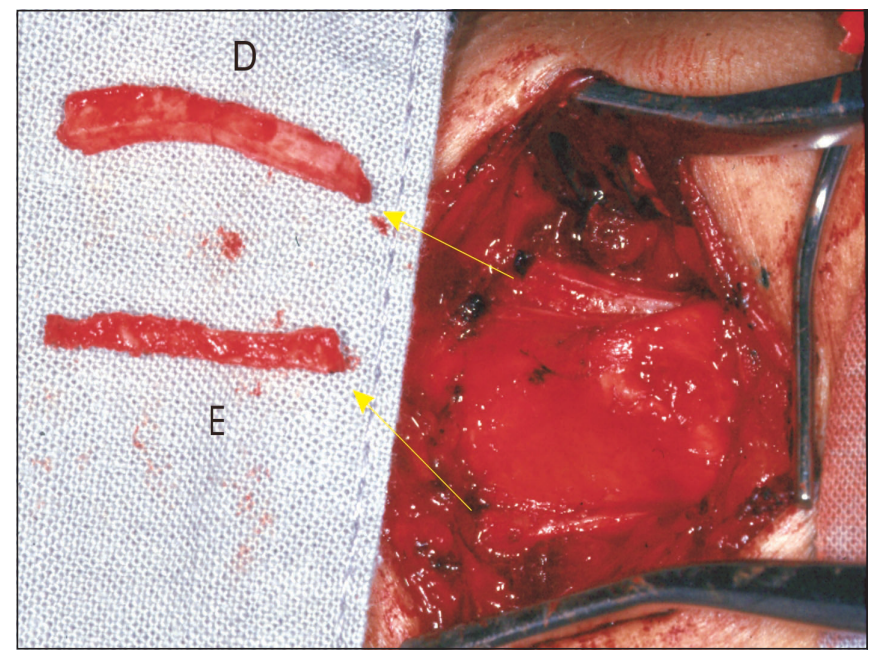

Figura 4. Uma nova fita é removida da lâmina da cartilagem tireóidea contralateral.

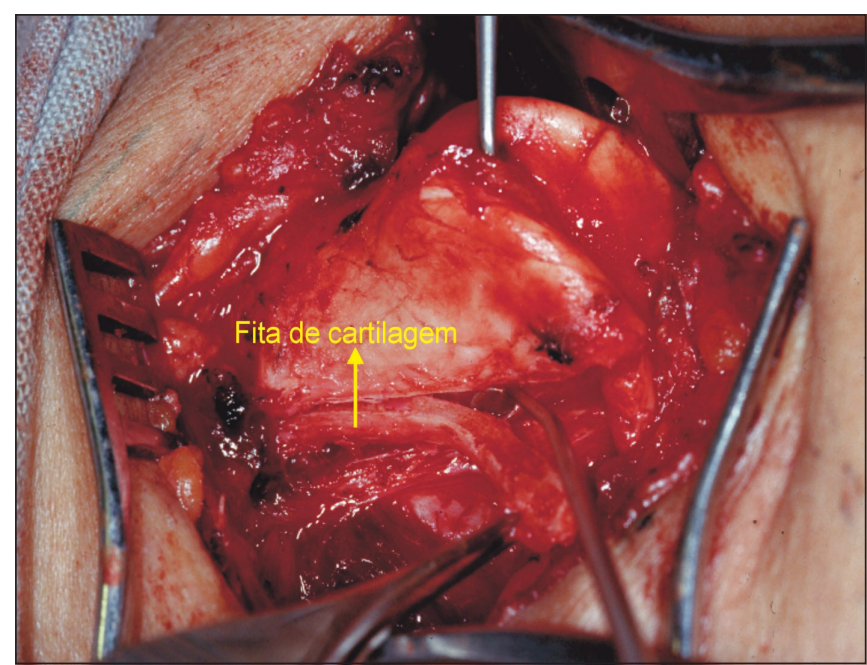

Figura 3. A fita de cartilagem é removida, preservando-se o pericôndrio interno.

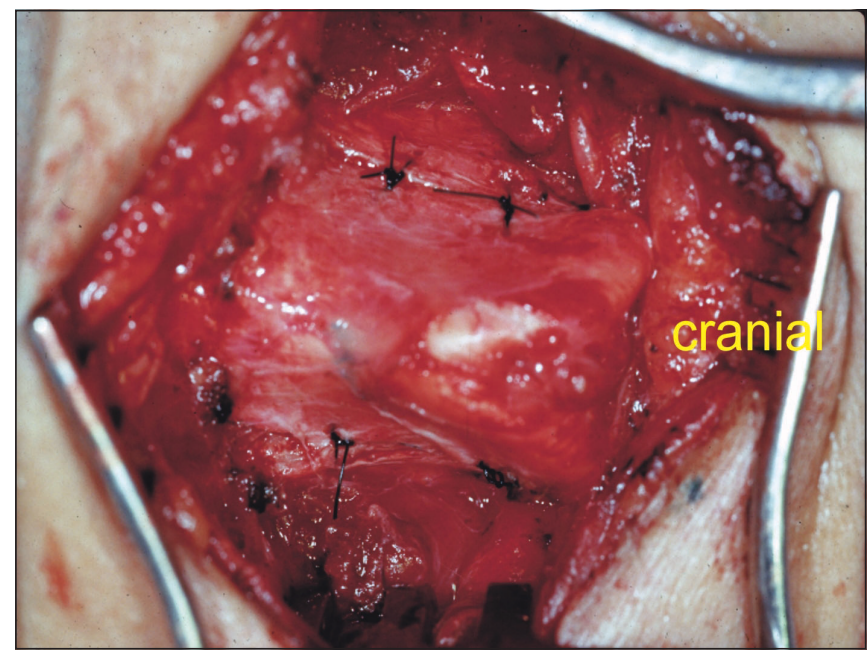

Figura 5. As bordas das incisões na cartilagem, de ambos os lados, são suturadas borda a borda.

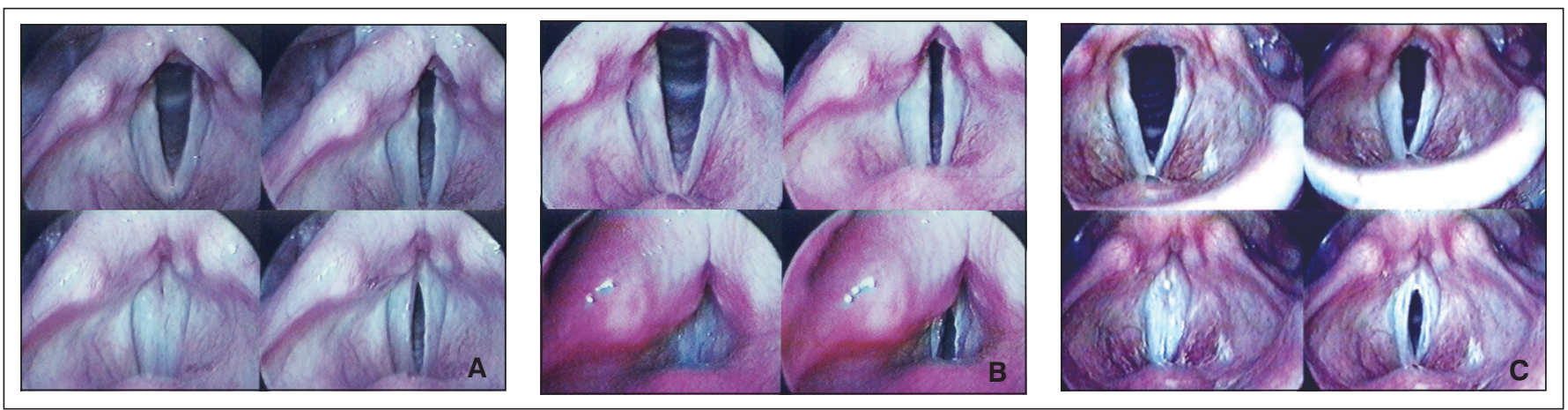

Figura 6. (a) Paciente dois, durante emissão vocal confortável com características de falsete - note aparente ausência de onda mucosa. (b) Presença de constrição supraglótica, com aproximação ântero-posterior das estruturas, observada durante tentativa frustrada de emissão em tom grave. (c) Videoestroboscopia no pós-operatório, durante emissão vocal em $136 \mathrm{~Hz}$, mostrando vibração cordal normal e presença de onda mucosa, sem constrição de estruturas supraglóticas. 
ser poupado a todo custo, evitando-se com isso traumatizar os tecidos moles situados internamente à cartilagem (Figura 3). Feita a remoção da fita de cartilagem e aproximação das bordas da lâmina tireóidea, a voz deve ser testada solicitando-se ao paciente que produza sons de fala normal, respondendo às perguntas formuladas pelo cirurgião, e também que emita fonemas variados. Caso o pitch vocal não seja suficientemente grave e satisfatório, a mesma intervenção é realizada no lado contralateral (Figura 4). Se ainda assim a voz não apresentar diminuição de freqüência esperada, o médico deve ampliar a largura da fita removida (em cerca de $1 \mathrm{~mm}$ ) de um dos lados e, se necessário, também do contralateral, até que se atinja o resultado almejado.

Uma vez finalizada a remoção da cartilagem, as bordas das incisões são aproximadas borda a borda e suturadas com fio de náilon 2.0 ou 3.0 (Figura 5). Dois a três pontos em "U" de cada lado são suficientes para estabilizar as lâminas da cartilagem tireóidea. Os músculos pré-laríngeos são aproximados, fato que é seguido pela colocação de dreno no espaço subcutâneo. A ferida cirúrgica é fechada, por planos, até a pele.

Nas primeiras 24h-48h, o dreno é retirado e os pontos na pele, removidos no $7^{\circ}$ dia pós-operatório. É recomendado ao paciente repouso vocal, por pelo menos sete dias, uma vez que certo grau de edema e eventual hematoma na mucosa interna da laringe são esperados. Além desses fatores, tal repouso também ajuda na estabilização das cartilagens. A antibioticoterapia é recomendada por uma semana e a administração de antiinflamatório nos três primeiros dias é conveniente, a fim de abrandar os sintomas dolorosos.

\section{APRESENTAÇÃO DOS CASOS}

\section{Caso 1:}

Paciente R.H., sexo masculino, 34 anos de idade, cor branca, referia voz aguda desde a adolescência, o que fazia com que as pessoas o confundissem, por exemplo, como se fosse uma mulher ao telefone. Queixava-se de sofrer constrangimentos sociais por causa desse fato. $\mathrm{O}$ exame otorrinolaringológico mostrou total normalidade das estruturas examinadas. A videoestroboscopia de laringe revelou normalidade dos movimentos vibratórios com periodicidade dos ciclos, simetrias de amplitude e de fases, e movimentos mucoondulatórios simétricos e aparentemente normais. A avaliação perceptiva denotou pitch agudo, porém com características de registro modal e loudness claramente normal. A apreciação da emissão sustentada confortável do fonema /e/, feita por meio do estroboscópico Key, demonstrou freqüência fundamental de $160 \mathrm{~Hz}$. Foram realizadas seis sessões de fonoterapia, sem que se obtivesse resultado satisfatório. Diante desse fato, foi indicada a tireoplastia tipo III de Isshiki, sob anestesia local. Inicialmente, foi realizada a remoção de uma tira vertical de cartilagem, com $3 \mathrm{~mm}$ de largura, do lado esquerdo, com imediata redução da freqüência fundamental. Entretanto, como a mesma não estava totalmente a contento do paciente nem do médico, decidiu-se pela remoção do mesmo montante de cartilagem do lado direito, obtendo-se um resultado vocal satisfatório. As extremidades das cartilagens foram suturadas borda a borda, com fio de náilon 3.0. O paciente teve alta no dia seguinte, sendo submetido a novas sessões de terapia vocal a partir do 7ํ dia pós-operatório, cessando o tratamento após quatro delas. Passadas quatro semanas da cirurgia, a vOz mostrou-se estável, com freqüência de $104 \mathrm{~Hz}$.

\section{Caso 2:}

Paciente V.P., sexo masculino, 35 anos de idade, cor branca, com queixa de voz fina desde a infância, referia problemas sociais decorrentes desse fato, o qual gerava situações embaraçosas. A avaliação perceptivo-auditiva da voz falada habitual denotou pitch agudo, compatível com registro de falsete, e discreta redução da intensidade. A freqüência fundamental, constatada por análise acústica computadorizada da emissão vocal /e/ confortável foi de 198 Hz. A avaliação otorrinolaringológica não evidenciou nenhum tipo de alteração orgânica no nível do trato vocal. A visualização videoestroboscópica da laringe mostrou pregas vocais de mobilidade normal e ligeiro arqueamento das bordas livres, porém com fechamento completo da glote durante os ciclos vibratórios (Figura 6a). A vibração cordal revelou periodicidade dos ciclos, simetria de amplitudes e fases e movimentos mucoondulatórios preservados e regulares. Quando solicitado para emitir som grave durante a estroboscopia, notou-se constrição supraglótica, com aproximação ântero-posterior das estruturas, sem diminuição do pitch, que manteve as características de falsete (Figura 6b). Foram instituídas oito sessões de fonoterapia, sendo que, ao final das mesmas, o paciente era capaz de emitir voz mais grave, de características masculinas, porém não conseguia manter tais características por tempo prolongado, tornando o ato fonatório cansativo e desconfortante. Perante o quadro, o paciente aceitou o tratamento cirúrgico, sendo submetido à tireoplastia tipo III de Isshiki sob anestesia local e sedação endovenosa. Esta foi realizada nos dois lados da cartilagem, à semelhança do primeiro caso, com resultado vocal imediato no transoperatório. O paciente foi mantido em repouso vocal por uma semana, sendo subseqüentemente encaminhado para orientação fonoaudiológica. Após o término desta, foi constatada qualidade vocal de características masculinas, com pitch grave e loudness ligeiramente reduzido. A análise acústica mostrou freqüência fundamental de $136 \mathrm{~Hz}$. A videoestroboscopia acusou parâmetros vibratórios normais, sem a hiperconstrição supraglótica inicialmente observada (Figura 6c). 


\section{DISCUSSÃO}

A qualidade vocal de um indivíduo pode ser considerada como marcante característica pessoal. Entre os diversos parâmetros que a caracterizam, o pitch é o fator mais fortemente relacionado com o sexo da pessoa. Assim, espera-se que sujeitos do sexo masculino tenham uma voz com o pitch mais grave do que os do sexo feminino. Segundo Behlau, Tosi e Pontes ${ }^{2}$ a freqüência fundamental do homem adulto brasileiro é, em média, de 113 Hz. Quando há discrepâncias evidentes, essa situação costuma trazer inúmeras conseqüências, como dificuldades profissionais, no convívio social e até, compreensivamente, na auto-estima ${ }^{1}$.

O falsete mutacional é caracterizado pela persistência de uma qualidade sonora aguda (semelhante à das mulheres) após a fase de muda vocal masculina, que normalmente ocorre durante a puberdade ${ }^{11}$. Nessa fase, a laringe do homem sofre considerável crescimento e, como conseqüência, as pregas vocais adquirem maior comprimento e massa, resultando em diminuição do pitch em torno de uma oitava. Em alguns indivíduos, porém, devido a causas provavelmente psicoemocionais, a voz permanece aguda, similar à feminina, apesar de a laringe apresentar características morfológicas e biomecânicas potencialmente adequadas para a emissão vocal masculina ${ }^{1}$. Hammarberg ${ }^{3}$ e Prathanee ${ }^{12}$ salientam que existem diferentes tipos de desordens vocais mutacionais:

1) a voz permanece com características pré-púberes devido a distúrbios endocrinológicos, com natural retardo no desenvolvimento da laringe;

2) o falsete mutacional que ocorre pelo fato de o paciente rejeitar a evolução normal do desenvolvimento da voz mais grave, mantendo, assim, as características agudas pré-púberes;

3) a mutação incompleta, que se distingüe por um rebaixamento de 4 a 6 semitons em relação à voz pré-púbere, ficando o pitch em torno de $200 \mathrm{~Hz}$;

4) a mutação vocal basal, com a qual o indivíduo mantém uma voz mais grave do que aquela em que a freqüência se estabilizaria no final da fase de muda vocal;

5) a voz de muda pervertida, que ocorre em mulheres e distingüe-se por aspereza e pitch vocal baixo com atributos masculinos.

O pitch vocal elevado pode também ser causado por fatores orgânicos, tais como atrofia, sulco vocal e cicatrizes de mucosa ${ }^{9}$. Entretanto, nesses casos, a voz apresenta, além da característica aguda, outras alterações, como soprosidade, rouquidão, aspereza e tensão. Excluídas as causas orgânicas supracitadas, o tratamento do falsete mutacional ou da muda vocal incompleta tem sido primordialmente a terapia vocal. Entretanto, em algumas situações, ela pode não ser suficiente, ou, mesmo que o paciente consiga a emissão de pitch mais grave (como ocorreu com nosso segundo paciente), a manutenção da qualidade vocal pode ser muito difícil, ou, ainda, completamente inviável do ponto de vista prático. Isshiki ${ }^{6}$ refere que fatores orgânicos em indivíduos com pitch vocal muito alto não devem ser excluídos simplesmente por não terem sido detectados pelos métodos diagnósticos de que dispomos. Alterações, como rigidez de mucosas, e características estruturais, como massa, espessura e comprimento das pregas vocais, podem ser a razão de um pitch elevado, porém muito difíceis de serem quantificadas e determinadas conclusivamente. Como o pitch vocal depende desses fatores, a possibilidade dessas causas orgânicas nos pacientes em questão deve ser sempre considerada, ainda que motivos funcionais sejam geralmente predominantes. Devido a essas dificuldades diagnósticas, Isshiki propõe que o uso da expressão "desordem mutacional" seja evitado, a menos que haja dados definitivos capazes de excluir fatores orgânicos. Sugere, ainda, que seja substituída por enunciações como "síndrome do pitch vocal muito alto" ou "voz masculina de pitch muito alto".

$\mathrm{Na}$ literatura, a voz excessivamente aguda na fase adulta do indivíduo masculino é mais freqüentemente classificada como falsete mutacional. Entretanto, é importante ressaltar que nem sempre os homens com queixa de voz aguda nessa faixa etária apresentam efetivamente voz peculiar ao falsete. O primeiro paciente apresentado neste trabalho pode ser considerado um exemplo típico dessa situação. Ele exibia uma qualidade vocal aguda, porém com freqüência fundamental de 160 Hz com particularidades de emissão modal. Já o segundo mostrava características vocais que, por estimativa auditiva, eram perfeitamente compatíveis com o registro de falsete e freqüência fundamental de $198 \mathrm{~Hz}$. Essa constatação nos leva a concordar com Isshiki e nos faz supor que, realmente, alguns homens, apesar de ter a laringe com características masculinas, com proeminência laríngea acentuada, calcificação da cartilagem e dimensões adequadas, podem apresentar pregas vocais mecanicamente favoráveis à emissão vocal confortável em uma freqüência fundamental mais elevada do que a da maioria dos indivíduos de mesmo sexo e idade. Nesse caso, o problema vocal não seria funcional, mas anatômico. Caso essa hipótese esteja correta, é perfeitamente compreensível que a terapia vocal não surta o resultado esperado uma vez que a emissão em tom grave não seria compatível com a estrutura mecânica das pregas vocais, exigindo um esforço muscular excessivo não-fisiológico, impedindo, dessa forma, a automatização do processo fonatório. Seria o mesmo que tentar levar alguém do sexo feminino com uma freqüência fundamental em torno de $230 \mathrm{~Hz}$ a automatizar a emissão em $110 \mathrm{~Hz}$. Evidentemente um estudo mais aprofundado, com número maior de pacientes, é necessário para comprovar nossas considerações. Os 
dois indivíduos que apresentamos neste trabalho fazem parte de um grupo de cinco homens com pitch vocal muito alto atendidos no serviço de otorrinolaringologia do Hospital das Clínicas da USP, no ano de 1999. Curiosamente, os dois pacientes que necessitaram do tratamento cirúrgico apresentaram idades mais avançadas do que os que obtiveram resultados satisfatórios com terapia vocal. Esse fato também nos leva a conjeturar que, quanto mais tempo uma pessoa permanece com um distúrbio funcional, mais difícil é a correção deste com fonoterapia, provavelmente em decorrência de profunda assimilação do padrão fonatório inapropriado.

A tireoplastia tipo III, como descrito por Isshiki, pode ser considerada um procedimento muito eficiente no tratamento de pacientes com pitch vocal excessivamente elevado. Do ponto de vista técnico e baseados em nossa experiência, essa cirurgia foi por nós considerada a mais simples entre as tireoplastias e, se realizada com cuidado, conforme a técnica preconizada por Isshiki ${ }^{6,10}$, nenhuma complicação grave deve ser esperada. A possibilidade de ajuste vocal durante o intra-operatório garante um excelente resultado funcional.

Sugerimos que a retirada vertical da fita de cartilagem seja feita com cuidado, removendo-se inicialmente não mais do que $3 \mathrm{~mm}$ de um dos lados e, se necessário, do outro, em vez de aumentar o montante extraído de um único lado. Essa sugestão baseia-se no fato de que o encurtamento unilateral da prega vocal pode resultar em assimetria de tensão entre as pregas, levando à assimetria de fases de vibração entre si. Embora, do ponto de vista vocal, a assimetria de fases não leve a alterações vocais perceptíveis ${ }^{5}$, acreditamos que, quanto mais próximas da normalidade forem as características funcionais da laringe, melhor será a saúde vocal do indivíduo a longo prazo.

Segundo estudo recente realizado por $\mathrm{Li}^{11}$, a tireoplastia tipo III não leva a alterações de parâmetros vocais, como jitter, shimmer, altura e intensidade vocais. Em sua pesquisa, o autor observou 15 pacientes submetidos a tal cirurgia e constatou que os resultados vocais foram satisfatórios em todos, sem nenhum prejuízo para qualquer dos parâmetros estudados. Slavit e col. ${ }^{13}$ relatam dois casos tratados com a técnica da tireoplastia tipo III (ligeiramente diferente da técnica utilizada por nós) com resultados satisfatórios. Entretanto, revelam que em um dos pacientes foi necessária uma segunda intervenção, um ano após a primeira, devido à recidiva da voz aguda em níveis próximos aos da freqüência fundamental anterior à cirurgia inicial.

Embora nossa experiência seja pequena, os resultados por nós obtidos estão de acordo com os apresentados por outros autores. Esse fato, associado à simplicidade da técnica, leva a acreditar que esta cirurgia deve ser sempre considerada como uma excelente alternativa terapêutica para estes pacientes, quando a terapia vocal falha ou quando ela não pode ser realizada por algum motivo.

\section{COMENTÁRIOS FINAIS}

A tireoplastia tipo III de Isshiki foi aplicada com sucesso em dois pacientes com pitch vocal excessivamente elevado, fazendo com que sua freqüência fundamental atingisse índices compatíveis com a voz masculina normal. Nenhuma complicação ou conseqüência indesejável foram constatadas na evolução desses casos. Tal fato, em conjunto com os dados da literatura, nos faz concluir que essa cirurgia é uma excelente alternativa para os casos em que as pregas vocais se apresentam morfologicamente normais, mas para os quais apenas a fonoterapia não é suficiente.

\section{REFERÊNCIAS BIBILIOGRÁFICAS}

1. ARONSON, A.E. - In Aronson, A.E. Clinical voice disorders - An Interdisciplinary Approach. $2^{\text {nd }}$ ed. New York, Thieme Inc., 1985. p. 146-7.

2. BEHLAU, M.S.; TOSI, O.; PONTES, P.A.L. - Determinação da freqüência fundamental e suas variações em altura ("jitter") e intensidade ("shimmer"), para falantes do português brasileiro. ACTA AWHO., 4:5-9, 1985

3. HAMMARBERG, B. - Pitch and Quality Characteristics of Mutational Voice Disorders before and after Therapy. Folia phoniat., 39:204-16, 1987.

4. ISSHIKI, N. - Functional surgery of the larynx. Dept. of Otolaryngology, Kyoto University Medical School ed., Kyoto, 1977. 207p. [Special report presented at $78^{\text {th }}$ Congress of Japan Otolaryngology Society, Fukuoka, $1977]$.

5. ISSHIKI, N. - In ISSHIKI, N. Phonosurgery theory and practice, Tokyo, Springer-Verlag, 1989, 5-21.

6. ISSHIKI, N. - In ISSHIKI, N. Phonosurgery theory and practice, Tokyo, Springer-Verlag, 1989, 131-9.

7. ISSHIKI, N. - Progress in laryngeal framework surgery. Acta Otolaryngol., 120:120-7, 2000.

8. ISSHIKI, N.; MORITA, H.; OKAMURA, H.; HIRAMOTO, M. Thyroplasty as a new phonosurgical technique. Acta Otolaryngol (Stockh)., 78:451-7, 1974.

9. ISSHIKI, N.;TAIRA, T.; TANABE, M. - Surgical alteration of the vocal pitch. J. Otolaryngol., 12:335-40, 1983.

10. ISSHIKI, N.; TSUJI, D.H.; SENNES, L.U. - Tireoplastias. São Paulo, Fundação Otorrinolaringologia, 1999. 191 p.

11. LI, GUO-DONG; MU, L.; YANG, S. - Acoustic evaluation of Isshiki Type III Thyroplasty for treatment of mutational voice disorders. $J$ Laryngol Otol., 113:31-4, 1999.

12. PRATHANEE, B. - Mutational falsetto voices: voice therapy. J Med Assoc Thai., 79:388-93, 1996.

13. SLAVIT, D.H.; MARAGOS, N.E.; LIPTON, R.J. - Physiologic Assessment of Isshiki Type III Thyroplasty. Laryngoscope, 100:844-8, 1990. 